\title{
Los movimientos sociales latinoamericanos como nuevas formas de democracia
}

\author{
Latin American Social Movements as \\ New Types of Democracy
}

\author{
Julia Urabayen ${ }^{1}$ \\ Universidad de Navarra (España)
}

\author{
Jorge León Casero ${ }^{2}$ \\ Universidad de Zaragoza (España)
}

Recibido: 21-02-19

Aprobado: 20-06-19

\section{Resumen}

Este artículo analiza algunos aspectos relevantes de los movimientos sociales latinoamericanos. Para explicar en qué consisten y cómo resuelven el problema de la organización política se estudia, siguiendo a Zibechi, algunos movimientos de dos países: Brasil y Bolivia. En el primero, en los años 80 surgieron unos movimientos sociales rurales muy fuertes que han perdido parte de su papel político durante los años del gobierno progresista del Partido de los trabajadores.

\footnotetext{
${ }^{1}$ (jurabayen@unav.es) Licenciada y Doctora en Filosofía. Selección de publicaciones recientes: "At the margins of ideal cities. The dystopian drift of modern utopias". Sage Open, 2018, pp. 1-11; "Espacio, poder y gobernamentalidad. Arquitectura y urbanismo en la obra de Foucault". Anales del Instituto de Investigaciones Estéticas, n 112, 2018, pp. 181 - 212; "Sob os passos do pensar concreto: Bergson e Marcel". Compêndio Gabriel Marcel (Homenagem aos 90 anos de publicaçao do Diário Metafísico). Editorial Edunioeste, 2017. ISBN 978-85-7644-341-4, pp. 193-218 y "Amando al otro, respondiendo a su presencia. Reflexiones sobre el amor desde la filosofía de Marcel”. La esencia del amor, Tirant Humanidades, 2017, pp. 27 - 47.

ORCID: https://orcid.org/0000-0002-0409-6279.

2 (jleon@unizar.es) Arquitecto, Licenciado en Filosofía, Graduado en Derecho, Doctor en Historia del arte. Selección de publicaciones recientes: "The Postmetropolis is God. Notes for a Phenomenology of the Urban Spirit”, Topoi. An International Review of Philosophy, vol. 38, 2019. https://doi.org/10.1007/s11245-018-9590-0; Disciplines of the City, Nova Publishing, USA, 2019; Trilogía Metrópoli, Editorial siníndice, Logroño, 2018 y "Mapa de Riesgo Social de Zaragoza: Resultados y conclusiones", Ciudad y Territorio, Vol. L, No 197, otoño 2018.

ORCID: https://orcid.org/0000-0002-3723.
} 
En el segundo, aparecieron en las mismas fechas movimientos sociales urbanos que transformaron un país que se había propuesto realizar desde el gobierno una reforma político-económica antiliberal.

Palabras-clave: movimientos sociales, democracia, Raúl Zibechi, Brasil, Bolivia.

\begin{abstract}
This paper analyzes some prominent aspects of Latin American social movements. In order to explain what they are and how they solve the problem of political organization, following Zibechi, some movements of two countries are studied: Brazil and Bolivia. In the first, powerful rural social movements emerged during the 80', but they have lost part of their political role during the years of the progressive government of the Workers' Party. In the second, during the same period urban social movements emerged and transformed a country whose government has decided to carry out an antiliberal politicaleconomic reform.
\end{abstract}

Key-words: Social Movements, Democracy, Raúl Zibechi, Brasil, Bolivia.

En las últimas décadas, la democracia se ha convertido en un tópico de la reflexión política que ha estado unido a las experiencias de crisis que han puesto en jaque los pilares del Estado de bienestar, de la democracia representativaparlamentaria y del Estado de derecho. En este contexto, se han puesto en práctica formas democráticas más cercanas al pueblo y más participativas que han sido denominadas "democracias radicales". Estas propuestas han recibido dos tipos de crítica. La primera considera que esas opciones no son viables en los países de grandes dimensiones: una democracia directa solo se puede aplicar en comunidades pequeñas. Pretender que ese sea un modelo de gobernanza política válido para organizaciones de mayor tamaño sería una utopía.

La segunda crítica se centra en los supuestos peligros unidos al otorgamiento del poder al pueblo, ya que este no es un sujeto político maduro, activo y responsable, sino manipulable y voluble. En ambas objeciones se destacan los rasgos negativos de una agrupación que parece carecer de definición, capacidad de reflexionar y principios organizativos, al menos, para grandes territorios.

En este trabajo, se analizará el modo en el que algunos movimientos sociales latinoamericanos llevan a cabo sus proyectos de democracia para mostrar cómo responden a esas dos objeciones. Dado que el tema es muy amplio, abordaremos un período concreto (el surgimiento masivo de los movimientos sociales en diversos territorios latinoamericanos desde la última 
década del siglo XX hasta los primeros años del nuevo siglo) y tomaremos como casos de estudio tanto movimientos rurales como movimientos urbanos estudiados por Raúl Zibechi ${ }^{3}$. Desde esta perspectiva se verá que esas formas políticas son comunidades que existen y funcionan territorializando, es decir, convirtiendo el espacio en territorio por medio del antagonismo.

Por ello los objetivos del artículo son dos. En primer lugar, reflexionar sobre la posibilidad de una democracia diferente a la del modelo hegemónico en Occidente durante los últimos siglos. En segundo lugar, contribuir al mejor conocimiento de la obra de Zibechi, un autor poco estudiado por los filósofos políticos europeos y que ha dedicado gran parte de su obra al análisis de los movimientos sociales latinoamericanos. La metodología utilizada, como se deduce de los objetivos, es la propia de la filosofía política, que no es otra que la descripción y la reflexión sobre el tema de estudio. Por último, la hipótesis del trabajo es que Zibechi, aunque muestra claramente los problemas a los que se enfrentan los movimientos sociales latinoamericanos, demuestra que estos han hecho posible un nuevo tipo de democracia.

\section{Más allá de la democracia parlamentaria: las reconfiguraciones occidentales de la democracia en la segunda mitad del siglo XX}

Las raíces teóricas de los debates sobre la democracia, al menos en Europa, surgen unas décadas antes de la actual crisis económica y política. Con el inicio en este continente de la crisis de la ideología comunista y del socialismo de Estado a finales de los años 70 y comienzos de los 80, la democracia se fue convirtiendo progresivamente en un significante flotante cuyo significado intentaron sobredeterminar un amplio espectro de planteamientos sociopolíticos.

Además de los principales movimientos sociales identitarios (entre otros, el feminismo, el ecologismo y el postcolonialismo), las ideologías políticas más relevantes que todavía batallan por su significado podrían ser clasificadas, a groso modo, en seis grupos: demócratas liberales en sentido amplio (Rawls, Nozick, Dworkin, Rorty), demócratas comunitaristas (Taylor, McIntyre, Sanders), demócratas de la tercera vía (Beck, Giddens), demócratas radicales (Mouffe, Laclau, Rancière, Balibar) demócratas de la multitud (Negri \& Hardt, Virno) y, si es que algo así es siquiera posible, demócratas maoístas-(y/o)leninistas (Badiou, Zizek).

A excepción de las dos primeras posturas y de algunas de las formas de democracia radical (Mouffe y especialmente Balibar), el resto de propuestas consideran insuficiente y obsoleto el actual modo de organización institucional,

\footnotetext{
${ }^{3}$ El artículo no se basa en un trabajo de campo propio, sino que es una reflexión a partir del trabajo de campo y el marco conceptual aportados por Zibechi.
}

Araucaria. Revista Iberoamericana de Filosofia, Politica, Humanidades y Relaciones Internacionales, año $22, \mathrm{n}^{\circ} 43$. Primer semestre de 2020. Pp. 77-98. ISSN 1575-6823 e-ISSN 2340-2199 https://dx.doi.org/10.12795/araucaria.2020.i43.04 
normalmente parlamentario. Por su parte, las dos últimas posturas se disputan, además del significado de la democracia como significante flotante, el del comunismo como significante vacío.

Concretamente, la propuesta de Negri y Hardt enfatiza la necesidad de abandonar la ideología decimonónica de la autogestión como modelo de administración establecido desde la Comuna de París en favor de una "gobernanza continua" entre movimientos sociales y gobiernos políticos. En palabras del italiano, "el concepto de autogestión permitía, para una clase obrera profesional del siglo XIX, encontrar la organización técnica del trabajo, el momento de la participación y la decisión: estaríamos locos si recurriéramos ahora a esta categoría, porque ya no tiene significado ante las figuras (políticas globales y financieras) de la producción contemporánea" (Negri 2007: 114).

Este "éxodo" de las instituciones públicas en favor de una gobernanza de izquierdas es criticado por Zizek por ser el proceder de una nueva ola de "comunistas liberales" indistinguibles de empresarios como Bill Gates o George Soros, a los que presenta como "enemigo[s] de cualquier lucha progresista" (Zizek 2013: 52). Siguiendo el "perspicaz informe" elaborado por Malnuit, Zizek comenta los diez mandamientos de los comunistas liberales: 1. Debes dar todo gratis (libre acceso, sin derechos de autor. Debes hacerte cargo de estos servicios adicionales, los cuales te harán aún más rico). 2. Debes cambiar el mundo, no solo vender cosas: la revolución global incluye la búsqueda de una sociedad mejor. 3. Debes compartir recursos, ser consciente de la responsabilidad social. 4. Debes ser creativo: presta atención al diseño, a las nuevas tecnologías y a las ciencias. 5. Debes decirlo todo: no debe haber secretos, apoya y practica el culto a la transparencia y a la libre circulación de información. 6. No debes trabajar nunca con horarios fijos: improvisa comunicaciones inteligentes, dinámicas y flexibles. 7. Debes volver a la escuela: comprométete con la educación permanente. 8. Debes actuar como una enzima: trabajar no solo para el mercado, sino también fomentar nuevas formas de colaboración social. 9. Debes morir pobre: devuelve tu fortuna a aquellos que la necesitan ya que, de hecho, tienes más de lo que puedes gastar. 10. Apoya al Estado: practica la asociación entre las empresas y el Estado. Es decir, practica una gobernanza continua en lugar de una autogestión autónoma y antagónica (Malnuit 2006: 32-37).

Frente a esta postura, Zizek opta por volver al eje pueblo-movimientopartido-líder (Zizek 2015), si bien lo hace sosteniendo una concepción no soberana del pueblo, no espontánea del movimiento, leninista del partido como organizador de los movimientos, y dotando al líder de un papel unificador entre partido y pueblo (Zizek 2003; Zizek 2004). Como premisa de todo ello, subyace el psicoanalítico y lacaniano postulado según el cual, en realidad, "la gente no sabe lo que quiere" (Zizek 2015: 1085). A pesar de que la propuesta 
"representativa" de Zizek va más allá de las instituciones de representación parlamentarias, es -al igual que la razón populista de Laclau a la que combateincapaz de servir de modelo conceptual a la hora de describir el modo de funcionamiento de los movimientos latinoamericanos.

Esos modelos democráticos han sido ignorados por la mayoría de las reconfiguraciones occidentales y cuando han sido tenidos en cuenta, han sido desechados. Hardt y Negri citan explícitamente los análisis de Zibechi a propósito de la comunidad aymara de El Alto (Negri y Hardt 2011), pero en última instancia rechazan su proyecto de autonomía autogestionada $\mathrm{y}$ fuertemente territorializada.

Si esto es así, los dos discursos occidentales más importantes que intentan suturar el significante democracia a través de una redefinición del proyecto comunista son incapaces de dar razón de los movimientos sociales latinoamericanos. Pensar esa forma de acción que no sigue los modelos de lo que Fanon llamó "la zona del ser" (Fanon 2010) requiere, por tanto, un marco teórico diferente.

\section{Más allá del nomos de la tierra: los movimientos sociales en América Latina}

Ese marco, así como el estudio de la situación concreta de los movimientos sociales latinoamericanos, se encuentra en la obra de Zibechi. Su análisis muestra que los movimientos son opciones políticas democráticas diferentes a las reconfiguraciones occidentales enumeradas en el apartado anterior. En esta sección se explicará cómo gracias a una reflexión atenta a los casos concretos, el autor ha ido matizando sus afirmaciones iniciales acerca de estas formas de acción política.

En sus primeros trabajos, Zibechi sostenía que los movimientos son: 1. Anticapitalistas, pues sus valores son totalmente diferentes a la competencia y el libre mercado. 2. En constante cambio e insurrección, ya que favorecen el proceso de creación de muchos mundos diversos y en continua transformación. 3. Antiestatales, dado que sus formas de organización son participativas y comunitarias (Zibechi 2007).

Estos rasgos implican, en primer lugar, que los movimientos no son los sindicatos ni los partidos políticos (Zibechi 2011). Son los sujetos colectivos que han ocupado el espacio de la resolución de los conflictos sociales que había sido monopolizado previamente por esos agentes (Zibechi 2018). En segundo lugar, estas características significan que los movimientos son una articulación de pueblo-masa-nación, que posee una gran capacidad de organizarse. Esta imbricación parte de una situación muy concreta: los Estados nación latinoamericanos se han establecido sobre la base de la exclusión y las relaciones 
sociales heterogéneas en las que, además, el control del trabajo no sigue un único patrón. De ahí que lo que se denomina genéricamente "movimiento social" sea llamado por sus agentes "naciones" "clases", "sectores populares" y "pueblos" (Zibechi 2018).

Las variaciones en la denominación son fundamentales, ya que muestran una realidad cambiante. Son las relaciones sociales en su totalidad las que se están movilizando. A diferencia de otros estudiosos de los movimientos sociales, Zibechi destaca la dispersión frente a la organización ${ }^{4}$. Además afirma que conservar esa dispersión implica reforzar el papel de la autonomía de los movimientos respecto a otros agentes sociales. En este sentido, el uruguayo denuncia las políticas sociales del Estado de bienestar, pues las considera herramientas de control y modos paternalistas de acción que tratan a los pobres como sujetos pasivos. Por esta razón critica también la aplicación de políticas sociales por parte de ONGs, personas bienpensantes o partidos/ gobiernos progresistas (Zibechi 2012). El modo en el que trabajan todas estas instituciones -mediante técnicos, dominio del saber, mapeado de las zonas, estadísticas y diagnóstico participativo- crea las élites de la pobreza, así como una división entre los movimientos razonables y los radicales, que deben ser

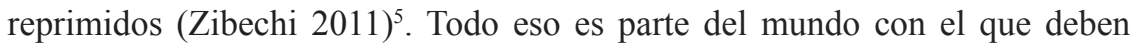
romper los movimientos sociales: el del control y el disciplinamiento por medio del subsidio (Zibechi 2010).

Las políticas sociales actuales usan las vías pacíficas que sostienen que el supremo arte de la guerra es someter al enemigo sin luchar. Estas medidas no militares o imperialismo blando fueron fomentadas por el Banco Mundial para luchar contra la pobreza bajo el supuesto de la conexión necesaria entre desarrollo y seguridad. Esta lucha, presentada bajo el ropaje ideológico de la participación de los pobres y la defensa de los derechos humanos, según Zibechi, lo que hace es dar a los pobres préstamos que provocan su endeudamiento perpetuo.

Ante estas medidas y organismos, los movimientos sociales tienen que aprender a preservar su autonomía y a tratar con las instituciones con las que mantienen una relación de desigualdad. Este es el mayor reto al que se siguen enfrentando actualmente. A lo que se une el problema de la cohabitación y la pérdida del espacio ya ganado. Para aclararlo se expondrá un único caso que es extrapolable a otras muchas ciudades latinoamericanas: el barrio Bello Oriente de la Comuna tres de Medellín. Como otras poblaciones surgidas de la miseria, esta tuvo que autoconstruirse. Tras ese duro trabajo, se ha tenido que enfrentar al proyecto Cinturón Verde de la ciudad que no solo pretende que no se siga

\footnotetext{
${ }^{4}$ García Linera indica tres componentes de los movimientos: estructura de movilización o toma de decisiones, identidad colectiva o registros culturales, y repertorios de movilización o mecanismos de lucha (García Linera 2004).

${ }^{5}$ Las organizaciones sociales no son los movimientos sociales; más bien los neutralizan (Zibechi 2011). Davis critica igualmente el papel que están jugando las ONGs (Davis 2006).
} 
construyendo en las laderas, sino que quiere desalojar a las familias que ocupan zonas que se desea "sanear" (Zibechi 2015). Este proyecto, como otros, supone la pérdida de un espacio convertido en territorio por la acción comunitaria.

Pero este no es el único frente: a esta pérdida de territorio como resultado de una intervención municipal, se une un antiguo problema que no se ha logrado resolver. Las bandas paramilitares que reclutan jóvenes y extorsionan a las comunidades. La situación de inseguridad que crean estas bandas ha permitido la militarización de los barrios que se quiere desalojar y que han sido sometidos a un alto control sin establecer la diferencia oportuna entre las acciones realizadas por los movimientos sociales y las ejecutadas por las bandas:

\begin{abstract}
“es cierta la irrupción -a raíz de los últimos treinta años de neoliberalismo- del fenómeno del narcotráfico, y también del tráfico de niños, mujeres, órganos... de mafias que lógicamente se han asentado en esos espacios donde el Estado es más débil, casualmente donde también se han asentado los movimientos. [...] en casi todos los países en los barrios populares tenemos mafias, delincuencias del modo más complejo, que compiten con los movimientos. En muchos casos agreden a los movimientos, terminan directamente destruyéndolos o generándoles enormes dificultades. Indirectamente, estas situaciones provocan la militarización de los espacios donde están los movimientos, que quedan debilitados al aparecer otros actores como narcos, paramilitares o iglesias evangélicas, además del Estado. [...] La situación para los movimientos en estos contextos provoca una dificultad enorme para poder seguir trabajando" (Zibechi 2012a: 192) ${ }^{6}$.
\end{abstract}

Así pues, las líneas que separan a los movimientos de otras formas de actuación política son a veces difusas y están en constante cambio al tener que resolver problemas diferentes que reclaman respuestas nuevas. Su carácter nómada-territorializante hace que su organización varíe sin por ello dejar de ser lo que son: acciones de sujetos políticos que actúan de manera antiestatal. Su multiplicidad no es, por tanto, un aspecto que indique agotamiento, sino justo lo contrario. Sin embargo, Zibechi dice que desde su nacimiento hasta hoy los obstáculos han sido numerosos y parecen haber debilitado seriamente a estas formas políticas (Zibechi 2018).

En 2016, el autor estudiado seguía manteniendo que es necesario reconstruir nuevos movimientos sociales y que eso iba a llevar mucho tiempo por la gran desmoralización y confusión existente (Zibechi 2016a). Por una parte, como ya se ha expuesto, esta situación se debe a los medios de domesticación que se han aplicado a los movimientos y, por otra, a la inexistencia de temas comunes que puedan aglutinar a la enorme cantidad de movimientos dispersos en numerosos

${ }^{6}$ El narcotráfico cumple unas funciones sociales y culturales concretas en la zona del no-ser: realiza el control social de quienes no pueden endeudarse (Zibechi 2017).

Araucaria. Revista Iberoamericana de Filosofia, Politica, Humanidades y Relaciones Internacionales, año $22, \mathrm{n}^{\circ} 43$. Primer semestre de 2020. Pp. 77-98. ISSN 1575-6823 e-ISSN 2340-2199 https://dx.doi.org/10.12795/araucaria.2020.i43.04 
países (Zibechi 2009). Es decir, la multiplicidad en este sentido puede generar algún problema.

El análisis realizado por Zibechi ha ido concediendo cada vez más peso a estas dificultades. En las publicaciones del año pasado sostuvo que en sus trabajos previos no captó adecuadamente la enorme capacidad de respuesta territorial de las políticas estatales, lo que le impidió ver que "las ganancias territoriales de los movimientos eran frágiles y transitorias, por lo menos en las condiciones de los movimientos urbanos" (Zibechi 2018: 7). Además, señaló que la dispersión actualmente es enorme. En Latinoamérica, hay movimientos muy fuertes que no son indígenas y la mayoría de ellos son organizaciones sociales, no movimientos: tienen jerarquías internas, división del trabajo, presupuestos fijos...

Estas características contrastan con el nacimiento de los movimientos, especialmente los indígenas, que surgieron de unas corrientes políticas de resistencia comunes: las comunidades eclesiales de base vinculadas a la teología de la liberación, la cosmovisión indígena que promovió un modo de entender las relaciones sociales diferentes al occidental -plasmadas en los conceptos de Sumak Kawsay y Suma Qamaña, que implican relaciones armoniosas entre los seres humanos así como entre estos y la naturaleza-, la educación popular y el guevarismo o compromiso ético con los de abajo (Zibechi 2014b).

Todos estos cambios le llevaron a sostener en 2012 que quizás el nombre "movimiento social" no era el más adecuado para referirse a estos modos plebeyos de hacer política. Se une una postura anticapitalista identificando tres rasgos de las dinámicas sociales características de América Latina: la vinculación con el territorio (la conversión, a lo largo del tiempo y a través del conflicto, del espacio en territorio), la tendencia a la autonomía (no identificación con los partidos, los sindicatos o las instituciones públicas) y la propensión a la horizontalidad o carácter antiestatal (Zibechi 2012a: 187). En 2018, este estudioso se mostró incluso más crítico con esa denominación, ya que "obtura la comprensión de prácticas colectivas diversas -siempre contrahegemónicas y en ocasiones anticapitalistas" (Zibechi 2018: 3).

Por ello en ese momento considera que hay que revisar las tres características que asoció inicialmente a estas formas de actuación política. La experiencia de los movimientos, especialmente de los rurales, de apropiación de la propiedad y gestión de los medios ha permitido ver que no todos los movimientos son anticapitalistas y que es necesario algo más que la propiedad y gestión colectiva para crear un mundo otro, pues en muchos casos "puede decirse que estamos ante una empresa capitalista similar a la que funcionaba con patrón" (Zibechi 2018: 8).

A la luz de esas experiencias, también es necesario reconsiderar la autonomía. En sus últimos trabajos, Zibechi estima que los movimientos 
funcionan más como anti-partidos que como anti-Estado. La relación que muchos movimientos han mantenido con los gobiernos progresistas y los partidos de izquierda han mostrado su debilidad intrínseca. Pero esta debilidad no ha afectado a todos y la autonomía continúa siendo el ideal. Lo que se ha aprendido es que ese ideal solo se cumple si involucra todos los ámbitos de la vida y no solo la producción. Por último, Zibechi examina de nuevo el papel que otorgó a la horizontalidad en la producción y matiza: este rasgo se da en las comunidades de base, pero coexiste con estructuras que se ocupan de otras tareas de forma jerárquica (Zibechi 2018). A pesar de esa puntualización, continúa afirmando que, si el movimiento no quiere despolitizarse, la jerarquía debe someterse a la base horizontal.

Es decir, Zibechi duda sobre la conveniencia del término "movimientos sociales", pero no sobre la existencia de los mismos; matiza su posición inicial, pero mantiene dos rasgos "básicos": territorialización o cambio continuo y autonomía. A lo que, en muchos casos, se une el aspecto anticapitalista. Este nuevo balance-ajuste tiene en cuenta la trayectoria de más de tres décadas. Al período álgido de nacimiento y creación de los movimientos, ha seguido otro marcado por cambios muy importantes en los países latinoamericanos: el ascenso al poder de los gobiernos de izquierda y, actualmente, la sustitución en el poder de esos partidos por otros de derechas. Esos cambios políticos han afectado profundamente a los movimientos sociales y la nueva situación política en la región está dando lugar a un nuevo ciclo con movimientos dotados de otros rasgos (como el carácter anticolonial) o con esos rasgos modulados de otra forma (Zibechi 2018). En todo caso, es necesario un lapso temporal mayor para establecer un balance fiable-definitivo.

Con el objetivo de clarificar qué son esos movimientos sociales durante el primer ciclo (el más documentado y el que analiza más extensamente Zibechi), veremos en el siguiente apartado las opciones políticas de los que optaron por no salir de las áreas rurales. El país elegido como referente será Brasil. Después analizaremos algunas de las formas en las que se dispersa el poder y se crean los movimientos como poderes no estatales en las zonas suburbanas de Bolivia. En ambas situaciones, es patente que el modo de actuar de los movimientos consiste en la formación de relaciones sociales en paralelo a las estatales, lo que crea "microsociedades más o menos integrales en las cuales hay espacio de poder, de producción, de educación y en algunos casos de sanidad" (Zibechi 2012a: 187$)^{7}$.

${ }^{7}$ La educación es un aspecto fundamental y es diferente según los movimientos: educación popular, irrupción de pueblos indígenas y negros que inciden más en la pedagogía del aprendizaje, y las comunidades en resistencia (Zibechi 2018).

Araucaria. Revista Iberoamericana de Filosofia, Politica, Humanidades y Relaciones Internacionales, año $22, \mathrm{n}^{\circ} 43$. Primer semestre de 2020. Pp. 77-98. ISSN 1575-6823 e-ISSN 2340-2199 https://dx.doi.org/10.12795/araucaria.2020.i43.04 


\section{Reterritorializaciones nómadas 1: la movilización rural en Brasil}

El análisis de los movimientos sociales rurales tomará como referencia uno de los más consolidados, de más larga trayectoria y que ha obtenido más resultados: el Movimiento de los Trabajadores Rurales Sin Tierra (MST), que se fundó en 1984. El MST nació a partir de diferentes agrupaciones previas, tales como la Comisión Pastoral de la Tierra, la teología de la liberación, y otros movimientos que tomaron cuerpo en otros períodos de la historia de Brasil, de cuya experiencia el MST extrajo una serie de principios que afectan a la vida comunal: educación, organización, producción y cooperación agrícola, ocupación, solidaridad y desarrollo, mística, marcha y reforma agraria (Zibechi 1998).

El contexto en el que surgió fue una realidad social de explotación, resultado de una política agraria latifundista en la que las grandes corporaciones internacionales devastaron los recursos naturales del enorme país americano y de una grave crisis económica que dejó a millones de personas sin tierra, pero sin opciones de partir hacia las ciudades -donde sabían que solo les esperaban las favelas- ni deseos de aceptar las políticas de desplazamiento de las poblaciones o la transformación de los sin tierra en mano de obra barata del garimpo (Mançano Fernandes y Stédile 2002).

En lo lugar de un éxodo, lo que se produjo fue un movimiento que se alzó y realizó diferentes acciones para reclamar una reforma agraria y especialmente una modificación del modelo neoliberal brasileño. Este movimiento logró implicar a familias enteras y a comunidades que se unieron para ocupar la tierra mediante asentamientos (fincas que han sido ocupadas y el Estado reconoce después como propiedad de quienes las han ocupado) y campamentos (terrenos ocupados cuya propiedad todavía no ha sido reconocida) ${ }^{8}$, así como para marchar en protesta hacia las ciudades, especialmente hacia la capital, reclamando cambios en la legislación y en la política (Mançano Fernandes y Stédile 2002).

Estas comunidades rurales se agruparon para crear un modo de trabajo no capitalista basado en la solidaridad, en el que no hay prácticamente división de tareas ni separación entre el que piensa y el que hace, ni imposición de plazos ni modos de organización. Los resultados han sido muy positivos, pues se han creado numerosas cooperativas (Zibechi 1998). Además, a estas iniciativas rurales, se unió la población de las ciudades, lo que convirtió al movimiento en una gran fuerza espontánea, es decir, no dirigida ni creada externamente 9 . Su fortaleza se debe a que es un movimiento popular que está abierto a la participación de todos. Por ello Zibechi lo considera un movimiento de movimientos (Zibechi 1998).

\footnotetext{
${ }^{8}$ Eso sucedió primero en los territorios rurales, luego en los urbanos y finalmente en los rururbanos (Zibechi 2012).

${ }_{9}$ Para Zibechi en los movimientos latinoamericanos no hay líder (Zibechi 2007).
} 
Además, es un movimiento social dotado de "tres características complementarias: sindical, popular y política" (Sergio y Stédile 1993). Es decir, posee unos rasgos que la mayoría de los movimientos no tienen tan definidos. La articulación de esas tres dimensiones se refuerza con unos principios organizativos claramente establecidos: la dirección colectiva o colegiada; la división de las tareas; la disciplina, que permite aceptar las decisiones colegiadas; el estudio y formación de cuadros; el rechazo de la cooptación o introducción en el movimiento de líderes corruptibles; y el vínculo con la base (Zibechi 2012a).

Este movimiento, por tanto, debe nutrirse de la experiencia de sus miembros, pero también formarse y reflexionar (Mançano Fernandes y Stédile 2002). En este caso, el estudio teórico fue posterior a su experiencia política y se centró en Lenin, Marx, Engels, Mao Tse Tung y Luxemburgo, así como en los pensadores brasileños y en los revolucionarios latinoamericanos. Por ser un movimiento rural siempre ha defendido la recuperación de los valores nacionales de Brasil así como la importancia de las áreas y las formas de vida rurales frente a las urbanas ${ }^{10}$.

Ahora bien, el MST no solo es un claro exponente de ese modo de hacer política de los de abajo, sino que también es un movimiento que tiene lugar en la mayor potencia latinoamericana y uno de los que apuesta por colaborar con el gobierno (Mançano Fernandes y Stédile 2002). De ahí que en su análisis, Zibechi preste atención a todos estos aspectos. En 2012, publicó un libro dedicado al gran gigante y en él sostuvo que "Brasil es uno de los pocos países del mundo que está escapando de la periferia" (Zibechi 2013: 7), lo que supone una serie de problemas que afectan a sus movimientos sociales.

El primero de ellos es el riesgo de convertirse en una potencia imperialista en la región. En 2004, Zibechi señaló que Argentina y Brasil tenían ante sí la opción de promover economías que reforzaran la exportación y la recaudación de impuestos con el fin de lograr una bonanza económica o virar hacia una economía más atenta a las necesidades de sus pueblos. La primera opción, que es por la que Brasil optó, supone una acumulación por desposesión (Harvey 2005), que genera una situación económica que ahoga a los países latinoamericanos, pues la política extractivista ya no necesita obreros para la producción ni para el consumo. La producción se realiza con máquinas automatizadas y robots, y las commodities se venden en países lejanos (Zibechi 2010a).

La apuesta brasileña por una política extractivista queda reflejada, por ejemplo, en los proyectos que forman parte de la IIRSA (Integración de la Infraestructura Regional Sudamericana) que han sido realizados con capital brasileño y ejecutados por empresas de ese país (Zibechi 2006) ${ }^{11}$. En todos esos proyectos, el impacto

${ }^{10}$ Esta mirada hacia la propia historia intelectual y social es normal, ya que la tradición de lucha por la tierra nace con la Conquista de América: los quilombos y los palenques (Zibechi 1998).

${ }^{11}$ En 2015, el uruguayo hizo un balance de ese proyecto (Zibechi 2015a). 
ecológico es inmenso y el objetivo principal es crear mejores conexiones para favorecer el comercio ${ }^{12}$. Esos proyectos -junto a las medidas económicas adoptadas por el gobierno siguiendo y potenciando los consejos del FMI y el BM- han permitido a Brasil alcanzar una posición dominante en América Latina. El país se ha convertido en potencia y lo ha hecho aumentando las desigualdades, y sin contar con los movimientos sociales ni con la sociedad civil (Zibechi 2013) ${ }^{13}$.

El segundo problema es justamente la pérdida de capacidad de actuación de los movimientos. Este es un fenómeno más amplio, ya que "todos los gobiernos progresistas han debilitado a los movimientos, algunos directamente, otros sin proponérselo, pero en los hechos los han debilitado" (Zibechi 2017a: 2). Esta pérdida es especialmente patente en Brasil, pues el crecimiento económico que lo ha convertido en potencia ha coincidido con el gobierno de Lula. Es decir, se ha producido durante uno de los gobiernos progresistas que quieren legitimarse sosteniendo que son la expresión de esos movimientos sociales que se levantaron contra las políticas neoliberales.

Ahora bien, la línea gubernamental Lula-Rousseff defiende una alianza Estado-capital privado brasileño y una noción de "capitalismo ético" que no otorga a los movimientos sociales un papel político relevante (Zibechi 2013). Por ello la política financiera de Lula continúa la línea neoliberal y ha quedado atrapada entre el FMI, el BM y las políticas neoliberales (Zibechi 2004a).

El gobierno progresista del Partido de los trabajadores sigue fomentando una economía extractivista que pretende compensar/frenar con el aumento de políticas sociales (Machado y Zibechi, 2016), especialmente en la modalidad de apoyo estatal a los proyectos socio-productivos o economía solidaria que fomenta la participación propia del modelo cooperativista al que subvenciona con recursos y personal especializado, lo que se ha convertido en "el mejor modo de invertir con eficiencia y más probable retorno de los recursos siempre escasos con los que cuentan" (Zibechi 2010a: 12).

En este período, los movimientos sociales, al menos algunos de los más importantes, han mantenido una cooperación-relación con el gobierno. El MST, como ya se ha señalado, ha aceptado la negociación con el gobierno de Lula como parte de su estrategia ${ }^{14}$. Por tanto, se encuentra en un equilibrio inestable: conserva su apoyo al gobierno, a pesar de que se ha distanciado porque este fomenta el agronegocio así como la alianza con las multinacionales y los empresarios de caña de azúcar para etanol, y, a la vez, recibe muchos fondos estatales dedicados a los asentamientos y la educación (Zibechi 2009).

\footnotetext{
${ }^{12}$ Como ejemplo puede tomarse el gasoducto Coari-Manaos. (Zibechi 2006). Davis expone el impacto medioambiental en las zonas urbanas hiperdegradadas y destaca diversos factores climáticos y humanos que han mermado las condiciones sanitarias de sus habitantes (Davis 2006).

13 Posteriormente Zibechi es más comedido (Zibechi 2017).

${ }^{14}$ Las relaciones con los partidos y el Estado son inevitables, pero lo importante es mantener la autonomía, lo que requiere una estrategia a largo plazo y unos sujetos colectivos bien consolidados (Zibechi 2017).
} 
Para Zibechi la cuestión clave es que esta forma de actuación gubernamental es, por una parte, un modo de controlar y domesticar los movimientos sociales debilitando su fuerza insurgente sin recurrir a la violencia, gracias a las negociaciones y los acuerdos $\mathrm{y}$, por otra, una transformación de los movimientos en sindicatos (Zibechi 2013). Por ello, al analizar la situación de Brasil bajo el gobierno del Partido de los trabajadores, Zibechi acepta el concepto de "hegemonía al revés" acuñado por Francisco de Oliveira ${ }^{15}$, a partir-invirtiendo a Gramsci. Los pilares de esta hegemonía al revés son tres: la dilución del conflicto, el uso de las políticas sociales para la cooptación y neutralización de los movimientos al despolitizar la pobreza y convertir la desigualdad en un problema técnico, y la aceptación por parte de los dominantes de ser "políticamente conducidos por los dominados, a condición de que 'la dirección moral' no cuestione la forma de explotación capitalista" (De Oliveira, R. Braga y C. Ribek 2017: 27).

Zibechi, por su parte, señala que hay dos lecturas de este fenómeno: la de quienes afirman que este gobierno, como otros progresistas, ha supuesto un avance porque ha reducido los niveles de pobreza (cosa que él no niega) y la de quienes (donde se incluye él) sostienen que no ha habido cambios en la desigualdad ni reformas estructurales, sino desindustrialización y reprimarización de la economía, lo que no es ningún avance (Zibechi 2015b).

En general, Zibechi afirma que, tras años de gobiernos progresistas en Brasil, los movimientos sociales han perdido su energía (Zibechi 2013). Aunque en los últimos años han surgido resistencias internas y externas, los movimientos sociales en Brasil ya no son los agentes de conflicto ni de cambio que fueron unas décadas antes. Según Zibechi, los movimientos sociales brasileños conocen un repunte en 2003 y muestran su unidad creando, a iniciativa del MST, la Coordinadora de Movimientos Sociales (CMS) que tiene una clara conciencia de que el cambio solo puede venir de los de abajo, pero no poseen la fuerza de los movimientos del primer ciclo $^{16}$.

El tercer problema es precisamente el fin de los gobiernos progresistas ${ }^{17}$. Según Zibechi, esta situación afecta a toda la región sudamericana, pero es

15 El sociólogo brasileño acuñó ese término y después el de "el revés del revés" que pretende explicar el fenómeno de los regímenes políticos, como el de Lula en Brasil, "que al llegar al poder practican políticas que son el revés del mandato de clase recibido en las urnas" (Zibechi 2011a: 20).

${ }^{16}$ Estos movimientos no han tomado las calles y para recuperar el papel político de los movimientos hay que volver al conflicto (Zibechi 2010a).

${ }^{17}$ En 2015, Zibechi dijo que "el ciclo progresista latinoamericano se está terminando" y aclaró: "entiendo por progresismo aquellos gobiernos que han intentado cambios en lo que fue el Consenso de Washington, pero nunca aspiraron a trascender el capitalismo en su fase extractivista y financiera" (Zibechi 2015b:117-118). Gudynas acota geográfica y temporalmente los progresismos. Además, señala que, aunque alcanzaron el poder por medio del sistema electoral y han pretendido legitimarse por el apoyo popular, han debilitado la participación ciudadana y se han alejado de los actores sociales, han lesionado la división de poderes y adoptado en gran parte un formato extremadamente presidencialista, unido a un rechazo de la crítica. Por último, si bien lograron éxitos en el crecimiento económico y la reducción de la pobreza, no han sido revoluciones postcapitalistas y se han convertido en Estados compensadores que han reducido la justicia a justicia económica redistributiva y asistencialismo (Gudynas 2016). 
más intensa en Brasil (Zibechi 2015b). Los escándalos de corrupción que han afectado a Rousseff, así como a Lula, sirven como argumento contra las ideas que pretendían defender y han producido un enorme desgaste ético que ha salpicado a los movimientos ${ }^{18}$.

\section{Reterritorializaciones nómadas 2: los movimientos urbanos en Bolivia}

A la hora de exponer los movimientos sociales urbanos, Zibechi toma como referencia principal a Bolivia. En 2007, destacó especialmente la situación de este país, donde tras varios años de luchas indígenas y populares se había logrado un triunfo muy importante: el de los aymaras que han sido capaces de modificar la política de su país desde fuera del Estado. Es decir, esta movilización permite captar muy bien un rasgo fundamental: "los movimientos bolivianos nos muestran que es posible -y no sólo deseable-construir poderes no estatales. O sea: que no todo poder debe ser un órgano separado y por encima de la sociedad" (Zibechi 2007: 21).

Para comprender bien el significado de este logro, conviene recordar que muchas de las comunidades estudiadas por Zibechi son agrupaciones que han nacido en los márgenes de las grandes ciudades ${ }^{19}$, en esos lugares en los que se hacinan las personas que se han desplazado desde las zonas rurales en busca de nuevas oportunidades para quedar encerradas en emplazamientos sin luz, sin agua potable, ni servicios públicos básicos: colegios, centros de atención sanitaria, transporte, recogida de residuos, alcantarillado... Allí se amalgaman los auténticos parias urbanos, los condenados de las ciudades (Wacquant 2000; Wacquant 2014). Estos son, para Zibechi, los resultados palpables de las gobernanzas neoliberales que fomentando la libertad, la competencia y la competitividad han producido lo que Wacquant ha denominado, en otro contexto, "las cárceles de la miseria" (Wacquant 2008) ${ }^{20}$ en las que están atrapados "los seres humanos marginales", pues "con el modelo liberal una parte de la sociedad sobra" (Zibechi 2012a: 188).

Los movimientos sociales son precisamente las respuestas de estos seres marginales. El análisis de Zibechi pretende mostrar con ejemplos concretos qué política se puede hacer desde la pobreza y no sobre la pobreza, ni muchos menos a costa de la pobreza (negocio altamente lucrativo). A la hora de exponer cómo se constituyen esos sujetos políticos, Zibechi primero

${ }_{18}$ El progresismo hoy acepta que "la corrupción es endémica a los sistemas políticos y abandona la pretensión de erradicarla" (Gudynas 2016: 54).

19 Se puede encontrar mucha información sobre estas ciudades, así como sobre otras en África y Asia en (Davis 2006). Para un estudio detenido de la teoría política vinculada a esa forma de urbanismo que segrega espacialmente, puede consultarse (Harvey 2016). Para información sobre la globalización y los guetos de algunas ciudades, véase (Harvey 2000).

${ }^{20}$ El libro estudia el sistema penal estadounidense, pero la noción “cárceles de la miseria" puede ser usada para hablar de esas zonas en las que los pobres quedan igualmente encerrados. 
desmonta un tópico: la conexión entre pobreza y violencia (Zibechi 2012). Los movimientos, como se ha dicho antes, comparten los espacios degradados con otros grupos, pero, a diferencia de ellos, crean comunidad al actuar juntos, como sucedió en El Alto, donde, debido a la ausencia de servicios públicos, la comunidad comenzó a desarrollar un tipo de organización antagónica e independiente del poder público institucionalmente sancionado, completamente autónomo y autogestionado, no representativo, caracterizado por procesos de democracia directa en la toma de decisiones y sin ningún tipo de relación con los distintos gobiernos territoriales. Se creó, por tanto, un modo de democracia sin una "gobernanza continua" entre movimientos y gobiernos (Zibechi 2007).

Los movimientos han creado comunidad, que en este caso se basa, en parte, en sus raíces ancestrales, y han atacado el modelo neoliberal y deslegitimado el sistema democrático tradicional que funciona a través de los partidos políticos, las elecciones y la representatividad parlamentaria. De este modo han dado lugar a un "mundo otro", un mundo en el que los instrumentos para generar las relaciones sociales de emancipación no son los Estados ni sus instituciones. Al hacerlo, han roto los tejidos del control social y han hecho patentes posibilidades de actuar que quedan ocultas en los momentos de reposo.

Estas formas de organización actúan horizontalmente, de forma espontánea o sin dirección y, basándose, en muchos casos, en la organización básica del parentesco. Las acciones de los movimientos introducen dispersión y deslegitiman el poder estatal (Zibechi 2007). Lo más importante en este punto es entender que las formas de organización, aunque son plurales, cuentan con ciertos principios: una noción diferente de propiedad, la deliberación colectiva y la rotación de la representación, que no es libre, sino un servicio obligatorio a la comunidad (Zibechi 2007).

Zibechi destaca, por tanto, que estos movimientos son en muchos casos comunidades nuevas que han creado espacios sociales y públicos en los que los vecinos se han hecho cargo de lo común, cada uno a su manera, pero todos con organizaciones políticas y económicas no capitalistas, no neoliberales y no parlamentarias. La clave de la organización política son las juntas vecinales, un poder que no es diferente a la sociedad, y "regula la construcción del barrio" (Zibechi 2007: 51-55) ${ }^{21}$. Es decir, se crece mediante una gestión colectiva de los recursos más importantes: el territorio, el comercio, la educación y la justicia. Así se cumplen las funciones del Estado sin el Estado: la decisión es siempre colectiva, se da rotación de dirigentes y tareas, y todo está descentralizado.

${ }^{21}$ La Ley de Participación de Bolivia impone algunos requisitos a esas juntas, lo que puede provocar una mayor separación respecto a la comunidad y un aumento de la corrupción. En otros casos, las juntas vecinales pueden tener menos funciones a su cargo o tener otras que son más urgentes en un momento concreto.

Araucaria. Revista Iberoamericana de Filosofia, Política, Humanidades y Relaciones Internacionales, año $22, \mathrm{n}^{\circ} 43$. Primer semestre de 2020. Pp. 77-98. ISSN 1575-6823 e-ISSN 2340-2199 https://dx.doi.org/10.12795/araucaria.2020.i43.04 
Las redes de colaboración poseen un fuerte carácter territorial establecido a través de su propia gestión urbanística y su organización de la producción. En lo referente a la primera, el urbanismo realiza un registro y gestión vecinal del suelo a través de planos de loteamiento que identifican la ubicación de cada predio, el número y propietario de cada lote, la emisión de títulos de propiedad del suelo ocupado e incluso el pago de impuestos vinculado a la propiedad de bienes inmuebles (Zibechi 2007).

Además, superando el mito de los comunes (Olstrom 2011)22, así como el de la nacionalización del suelo, en El Alto se instaura una propiedad individual (privada) de los lotes (si bien los puestos de venta son propiedad colectiva y los vendedores únicamente tienen título de poseedor), organizada como una red intrincada de calles en torno a un espacio público poli-funcional (la cancha) cuyo mantenimiento se realiza de forma colectiva y rotatoria, lo cual nos lleva a la organización de la producción (Zibechi 2012).

Esta última se estructura a través de una mínima división del trabajo en la que todos los trabajadores pueden rotar sin que el proceso productivo se resienta, favoreciendo de este modo la libre organización del tiempo del trabajador siempre que cumpla con los pedidos, sin que exista ningún tipo de vigilancia patronal (Zibechi 2007) ${ }^{23}$. Además, el trabajo cooperativo no capitalista se plantea desde la familia y otorgando un papel clave a las mujeres (Zibechi 2012) ${ }^{24}$.

Estas características de los movimientos, según Zibechi, son la condición de posibilidad de "una insurrección sin dirección ni dirigentes" (Zibechi 2007: 71) en la que la auto-organización es perfecta, tal y como quedó patente durante la "revolución del agua" del año 2000. Estas acciones negativas, al igual que las positivas descritas antes, han adoptado numerosas y variadas formas - desde la insurrección, la ocupación de tierras, la marcha sobre las grandes ciudades hasta el tener un gran número de hijos- y han dado lugar a un nuevo tipo de revolución que es diferente a las europeas.

Ahora bien, más allá de su potencial revolucionario, lo que aquí nos interesa destacar de la democracia propia de los movimientos urbanos bolivianos es que ha sido capaz de constituir una institucionalización antagónica plena que aúna los tres poderes clásicos de las teorías políticas ilustradas (legislativo, ejecutivo y judicial), que permanecen completamente independientes de

${ }^{22}$ Los debates sobre los bienes comunes no son debidos a la concesión del Nobel a Olstrom ni a la publicación de la obra de Negri y Hardt, sino que "hay una defensa de lo común ya que si el capitalismo consigue colonizar lo común, la vida se termina” (Zibechi 2012a: 190-191).

${ }^{23}$ A estos movimientos se une la idea de poder-Estado entre los aymaras, lo que incidirá en las formas de trabajo vinculadas al Ayllu: "las formas de organización del ayllu estarían basadas en cuatro formas de relación social del trabajo: ayni (cooperación entre familias del ayllu), mink'a (reciprocidad entre ayllus), mit'a (reciprocidad entre ayllus y la marka) y q'amaña (reciprocidad relacionada con el espacio ecológico)" (Zibechi 2007: 156).

24 Davis, por su parte, señala que es muy propio de la situación que se produjo en la década de los 70, en la que a las políticas del FMI se unió una crisis económica, cargar el peso de la subsistencia familiar sobre las mujeres y los niños (Davis 2006). En 2018, Zibechi reconoce que en sus trabajos previos subestimó el papel de los movimientos de mujeres y de los feminismos (Zibechi 2018). 
las instituciones oficiales reconocidas estatal e internacionalmente, y sin renunciar por ello a una completa autogestión basada en la democracia directa y asamblearia para la toma de decisiones colectivas.

Concretamente, el poder legislativo se ejerce a través de asambleas generales mensuales, el ejecutivo a través de una junta vecinal de carácter rotatorio que regula la edificación del barrio y los servicios mínimos, y el judicial a través de juntas vecinales para la resolución de conflictos ${ }^{25}$. Por su parte, la condición de ciudadanía se establece en función de la pertenencia a la junta vecinal como propietario de un lote otorgado por esta al vecino para la edificación de su vivienda. Todo ello está organizado en una red potencialmente infinita de juntas vecinales federadas cuyas dos únicas condiciones de acceso son poseer un plano de urbanización (ordenación del territorio) y no superar un máximo de 200 familias o mil vecinos, lo que garantiza la posibilidad de una autogestión organizada a través del ejercicio de la democracia directa.

Esta dispersión del poder es lo que dificulta el control social, la vigilancia y el panóptico. De ahí que las acciones externas se orienten contra esa característica de los movimientos buscando crear comunidades urbanas más grandes y, por ello, más fácilmente controlables, donde la inversión económica tenga mayores beneficios y el capitalismo pueda crecer junto a la democracia representativa. Este fue el principal punto de batalla identificado por la agencia estadounidense para el Desarrollo Internacional de Bolivia, que se propuso el objetivo de superar las organizaciones vecinales, "actualmente fragmentadas y atomizadas", para poder implementar "procesos de profundización democrática y responsabilidad ciudadana", articulándolas en barrios de entre 5000 y 8000 habitantes como "umbrales mínimos de vida en comunidades urbanas" (Zibechi 2007: 65-67).

Por tanto, lo que hace esta agencia, al igual que todas las tácticas propias del imperialismo blando características del neoliberalismo ${ }^{26}$, es volver a introducir el control -así como la gestión de la población y el territorio mediante el castigo de ciertas conductas (las que no colaboran o son violentas)-y forzar a las comunidades a endeudarse, lo que supone entrar en una óptica de trabajo y consumo capitalista.

Zibechi afirma que la dispersión propia de las comunidades implica que cualquier plan para fomentar su supuesto empoderamiento provoca un aumento de su vulnerabilidad. La situación de esas comunidades no se cambia fortaleciendo el poder y las formas de dominación, entre las que se encuentra la idea de que la democracia, la representativa, es el único modo de legitimar el poder. Como tampoco se logra por medio de las políticas implementadas por un gobierno progresista que no cuente con los movimientos.

${ }^{25}$ La resolución de conflictos funciona de diferente manera en las zonas rurales, en las que todos se conocen, y en las urbanas en las que los castigos son ejemplares y se ejecutan rápidamente.

${ }^{26}$ Zibechi dice que la solidaridad son los restos que da el que vive con comodidad y no encuentra un hueco para la acción política en el país en el que habita (Zibechi 2012).

Araucaria. Revista Iberoamericana de Filosofia, Política, Humanidades y Relaciones Internacionales, año $22, \mathrm{n}^{\circ} 43$. Primer semestre de 2020. Pp. 77-98. ISSN 1575-6823 e-ISSN 2340-2199 https://dx.doi.org/10.12795/araucaria.2020.i43.04 


\section{Más allá de las instituciones soberanas: balance de los movimientos sociales latinoamericanos}

A lo largo de estas páginas se ha expuesto, siguiendo a Zibechi, el sentido de los movimientos sociales que han tomado cuerpo en América Latina durante las últimas décadas. Con el propósito de lograr una mayor claridad se ha aceptado la diferencia entre el primer y el actual ciclo de los movimientos sociales, y se ha prestado una mayor atención al primer período. Además, se ha señalado que, al tomar en cuenta la experiencia y el contexto político de los últimos años, Zibechi comprendió la necesidad de matizar sus afirmaciones iniciales, que son las que se ajustan mejor a los movimientos presentados en las secciones dedicadas a Brasil y Bolivia.

Al reflexionar sobre la trayectoria de los movimientos, Zibechi señala que es necesario recordar que los movimientos "lucharon contra el modelo neoliberal, para frenarlo, para destruirlo, pero no necesariamente para colocar a la izquierda en el gobierno" (Zibechi 2016a: 11). Las formas de actuación de los movimientos, como se ha expuesto, son las de los de abajo y las de los Estados son las de los arriba. Por ello son antagónicas y toda acción estatal, aunque sea realizada por gobiernos progresistas, busca "hacer todo lo posible para que [los pobres] no consigan entrar en contacto con otros como ellos" (Zibechi 2011: 9).

De ahí que tanto los gobiernos neoliberales como los progresistas compartan la asunción de que el pobre es un sujeto pasivo de políticas estatales y no un sujeto político activo. Como es lógico, Zibechi establece diferencias en la manera en la que cada gobierno ha afrontado la relación con los movimientos sociales. Pero, según él, en todos los casos, los movimientos han sido debilitados por los gobiernos progresistas, no solo por los neoliberales. Esta es una afirmación que es un aspecto original de la postura de Zibechi frente a otras interpretaciones del mismo fenómeno político.

Por otra parte, actualmente se ha llegado al final de la etapa progresista sin que se haya podido establecer claramente el balance para el campo popular, que incluye a los movimientos. A pesar de esta situación sujeta a dificultades y cambios, Zibechi afirma que los movimientos latinoamericanos no han desaparecido y siguen sosteniendo que la solución a la pobreza es política, no técnica; comunitaria, no capitalista; participativa, no representativa. De ahí que enfrentarse a ella requiera la formación de sujetos políticos, lo que implica la creación dispersa de poderes sociales no estatales. Esta insistencia en la dispersión es otra de las aportaciones propias de Zibechi.

En este sentido, las políticas de los movimientos están sujetas a una de las críticas expuestas en la introducción: estas formas de hacer política funcionan solo en la escala micro, no en la macro, que está ya totalmente colonizada 
por el capital. Zibechi reconoce esta característica, pero afirma que no es una limitación, sino una oportunidad para cambiar la visión tradicional de la política: "probablemente a medio y largo plazo los macro Estados que conocemos no serían sustentables, y sí unidades más pequeñas, más dispersas. No debemos dar por sentado que en el largo plazo el Estado nación vaya a sobrevivir" (Zibechi 2012a: 191). De ahí que el tercer rasgo principal del análisis de Zibechi consista en destacar la capacidad que poseen los movimientos de actuar al margen de los partidos y el Estado.

La apuesta por los movimientos sociales es, por tanto, el reconocimiento de una reconfiguración antagónica de la democracia en la que la vida política es plural y crece de forma dispersa. Además, los movimientos se mantienen en su multiplicidad como contrapoderes que son antagónicos por definición, ya que el antagonismo es el que crea el espacio social. Esta visión de la política no es una utopía, sino una realidad no desorganizada -como se afirma en la segunda crítica señalada en la introducción-, que en la actualidad se encuentra en un momento muy delicado.

Tras unas décadas en las que se crearon multitud de movimientos sociales en América Latina y, tras afrontar diferentes problemas y retos, ahora estas formas de hacer política son realidades consolidadas que han aprendido que los cambios políticos son lentos y requieren "mucha paciencia, mucho trabajo de formación, educación, debate" (Zibechi 2012a: 193). Es decir, aunque en algunos lugares han sido debilitados, no han desparecido y siguen practicando una nueva forma de democracia. El análisis de Zibechi, por tanto, es autocrítico $\mathrm{y}, \sin$ ocultar las dificultades, ha rechazado que una democracia de otro tipo sea una utopía inalcanzable.

De lo expuesto en este artículo se puede concluir que los movimientos latinoamericanos llevan desarrollando durante más de tres décadas una democracia antagónica, no representativa y autogestionada que ha puesto en jaque no solo a los gobiernos y las instituciones, sino también a todas las teorías demócratas desarrolladas por Occidente durante el mismo lapso temporal. La democracia de los movimientos sociales latinoamericanos es, pues, el punto ciego de la filosofía política occidental. El excluido perverso que se niegan a reconocer como proyecto válido, a pesar de que exige ser discutido. Su misma persistencia supone, por el simple hecho de existir, la imposición por la fuerza de un desplazamiento obligado de la línea de lo políticamente posible: la apertura de una auténtica democracia à venir, hace ya tiempo que llegó, tal y como se ve en los movimientos sociales latinoamaricanos. 


\section{Referencias bibliográficas:}

Davis 2006: M. Davis, Planeta de ciudades miseria (Madrid, 2006).

De Oliveira, Braga y Ribek 2017: F. De Oliveira, R. Braga y C. Ribek, Hegemonias às avessas (Sao Paulo, 2010).

García Linera 2004: A. García Linera, Sociología de los movimientos sociales en Bolivia (La Paz, 2004).

Gudynas 2016: "Los progresismos sudamericanos: Ideas y prácticas, avances y límites" [en E. Gudynas, M. Svampa, D. Machado, A. Acosta y R. Zibechi: Rescatar la esperanza. Más allá del neoliberalismo y el progresismo, Barcelona, 2016],pp. 27-61.

Harvey 2000: D. Harvey, Spaces of Hope (Edinburgh, 2000).

Harvey 2005: D. Harvey, The New Imperialism (Oxford, 2005).

Harvey 2016: D. Harvey, Urbanismo y desigualdad social (Madrid, 2016).

Machado y Zibechi 2016: D. Machado y R. Zibechi, Cambiar el mundo desde arriba. Los límites del progresismo (La Paz, 2016).

Malnuit 2006: O. Malnuit, "Pourquoi les géants du business se prennent-ils pour Jésus?" en Technikart, Febrero (2006), pp. 32-37.

Mançano Fernades y Stédile 2002: B. Mançano Fernades y J. P. Stédile, Brava gente. El MTS y la lucha por la tierra en el Brasil (Barcelona, 2002).

Negri 2007: A. Negri, Goodbye Mr. Socialism (Barcelona, 2007).

Negri y Hardt 2011: A. Negri y M. Hardt, Common Wealth. El proyecto de una revolución del común (Madrid, 2011).

Olstrom 2011: E. Olstrom, El gobierno de los bienes comunes. La evolución de las instituciones de acción colectiva (México D. F., 2011).

Sergio y Stédile 1993: F. Sergio y J. P. Stédile, "A LUTA PELA terra no Brasil", Página Aberta, http://www.paginaaberta.com.br/ (1993), pp. 37-39.

Wacquant 2000: L. Wacquant, Parias urbanos. Marginalidad en la ciudad a comienzos del milenio (Buenos Aires, 2000).

Wacquant 2008: L. Wacquant, Las cárceles de la miseria (Buenos Aires, 2008).

Wacquant 2014: L. Wacquant, Los condenados de la ciudad, gueto, periferias y Estado (Madrid, 2014).

Zibechi 1998: R. Zibechi, "Brasil. El movimiento Sin Tierra. Una nueva vida en esta vida" en Viento sur, 39 (1998), pp. 7-16.

Zibechi 2002: R. Zibechi, "Argentina. Para producir no hacen falta patrones" en Viento Sur, 65 (2002), pp. 21-28.

Zibechi 2004: R. Zibechi, "El nuevo imperialismo y América Latina” en Viento Sur, 74 (2004a), pp. 45-49.

Zibechi 2004a: R. Zibechi, "Brasil. El gobierno Lula entre dos aguas" en Viento Sur, 76 (2004b), pp. 60-64. 
Zibechi 2005: R. Zibechi, Genealogía de la revuelta. Argentina (Bilbao, 2005). Zibechi 2006: R. Zibechi, "IIRSA: la integración a la medida de los mercados" en Ecología política, 31 (2006), pp. 19-25.

Zibechi 2007: R. Zibechi, Dispersar el poder. Los movimientos sociales como poderes antiestatales (Barcelona, 2007).

Zibechi 2009: R. Zibechi, "Gobiernos y movimientos: entre la autonomía y las nuevas formas de dominación" en Viento Sur, 100 (2009), pp. 247-254.

Zibechi 2010: R. Zibechi, Contrainsurgencia y miseria. Las políticas de combate a la pobreza en América Latina (México, 2010).

Zibechi 2010a: R. Zibechi, "Políticas sociales, gobiernos progresistas y movimientos antisistémicos" en RIFP, 35 (2010a), pp. 5-20.

Zibechi 2011: R. Zibechi, Política y miseria (Málaga, 2011).

Zibechi 2011a: R. Zibechi, "Ecuador. La construcción de un nuevo modelo de dominación” en Viento Sur, 116 (2011a), pp. 15-24.

Zibechi 2012: R. Zibechi, Territorios en resistencia. Cartografía política de las periferias (Málaga, 2012).

Zibechi 2012a: R. Zibechi, “Entrevista a Raúl Zibechi” en Papeles de relaciones ecosociales y cambio global, 118 (2012a), pp. 187-195.

Zibechi 2013: R. Zibechi. Brasil potencia. Entre la integración regional y un nuevo imperialismo (Lima, 2013).

Zibechi 2014: R. Zibechi, Descolonizar la rebeldía. (Des)colonialismo del pensamiento crítico y de las prácticas emancipatorias (Málaga, 2014).

Zibechi 2014a: R. Zibechi, "La révolution décolonisatrice du zapatisme" en Alternatives Sud, 21, 2 (2014a), pp. 85-108.

Zibechi 2014b: R. Zibechi, "Liderar el mundo que late en el corazón de los movimientos" en Kavilando, 6, 1 (2014b), pp. 7-14.

Zibechi 2015: R. Zibechi, "Medellín. La ladera grita, se resiste y construye" en Kavilando, 7, 1 (2015), pp. 39-46.

Zibechi 2015a: R. Zibechi, "Interconexión sin integración: 15 años de IIRSA" en El Comercio. 15 de septiembre de 2015 (2015a).

Zibechi 2015b: R. Zibechi, "Hacer balance del progresismo" en Kavilando, 7, 2 (2015b), pp. 117-120.

Zibechi 2016: R. Zibechi, Latiendo la resistencia. Mundos nuevos y guerra de despojo (Málaga, 2016).

Zibechi 2016a: R. Zibechi, "Situación en Sudamérica. Entrevista a Raúl Zibechi" en Libre Pensamiento, 88 (2016a), pp. 8-13.

Zibechi 2017: R. Zibechi, "Entrevista al periodista e investigador Raúl Zibechi: 'Venezuela podría convertirse en la Siria de América Latina"' en Resumen Latinoamericano, 1 de julio 2017 (2017), pp. 1-5. 
Zibechi 2017a: R. Zibechi, "Los sujetos de las oleadas revolucionarias son los movimientos, no los Estados" en http://kaosenlared.net/raul-zibechilos-sujetos-las-oleadas-revolucionarias-los-movimientos-no-los-estados/ (2017a).

Zibechi 2018: R. Zibechi, Movimientos sociales en América Latina. El "mundo otro" en movimiento (Málaga, 2018).

Zizek 2003: S. Zizek, A propósito de Lenin. Política y subjetividad en el capitalismo tardio (Buenos Aires, 2003).

Zizek 2004: S. Zizek, Repetir Lenin (Madrid, 2004).

Zizek 2013: S. Zizek, Sobre la violencia. Seis reflexiones marginales (Barcelona, 2013).

Zizek 2015: S. Zizek, Menos que Nada. Hegel y la sombra del materialismo dialéctico (Madrid, 2015). 